BNL - 68641

Informal Report

CAP-317-CK-R

An Investigation of the Beam Monitor for the Cluster Klystron

Yongxiang Zhao

Brookhaven National Laboratory

August 2001 
BNL - 68641

Informal Report

$C A P-317-C K-R$

An Investigation of the Beam Monitor for the Cluster Klystron

Yongxiang Zhao

Brookhaven National Laboratory

August 2001 


\title{
AN INVESTIGATION OF THE BEAM MONITOR FOR THE CLUSTER KLYSTRON ${ }^{1}$
}

\author{
Yongxiang Zhao \\ Physics Department, Bldg. 901-A \\ brookhaven national laboratory, Upton, NY 11973
}

\begin{abstract}
The cluster klystron project required a beam monitor to check the quality of the hollow beam shape. Since the power density of the beam is very large, a common phosphorescent screen doesn't work. We investigated varies types of monitors. The related problems were aslo discussed.
\end{abstract}

\section{THE ISSUE}

The cluster klystron project required to set up the MIG gun test. A big concern is the quality of the beam: can it pass through the drift tube with a designed ring shape - a good hollow shape beam. First of all, we have to have someway to monitor the profile of the beam. However, the beam current as well as the energy per bunch is pretty high. A normal screen will, most likely, be damaged. Thus a careful investigation is necessary.

Various methods have been investigated. Based on it some options were proposed. ${ }^{[1]}$ We considered the simplest way is to use a foil intercepting the beam and monitor its infra-red and/or visible light image. The material could be tungsten or tantalum, or carbon. Tantalum seems the best. The disadvantage is that the dynamic range is narrow. Thus if the beam energy is too high, the foil will be burnt. If it is too low, the temperature will be too low to show a clear image. The ratio of maximum to minimum is only 4 to 5 , and the level is not adjustable.

An X-ray image detected by a pin-hole camera is another option. The intensity is adequate to be detected and the dynamic range is only limited by the sensitivity of the camera. However, it needs an X-ray image intensifier which converts an X-ray image to a visible image. The resolution depends on the pin hole and is no better than twice of the diameter.

A phosphorescent film layered on the back of beryllium foil is another option. The luminescence is not due to the electrons, but stimulated by the X-rays produced by the electrons hitting the beryllium foil at the point just preceding the phosphorescent. This avoid the risk that direct bombardment will damage the phosphorescent layer.

Following is a list of the beam parameters, on which the quantitative estimate is based.

1. The cluster klystron project was terminated in 2001 due to the shortage of budget and other factors. This file is for documentation that may serve as a reference. 
Table 1: The beam parameters

\begin{tabular}{|c|c|c|c|}
\hline Parameter & Svmbol & Value & Unit \\
\hline Peak Voltage & $\mathrm{V}$ & 100 & $\mathrm{kV}$ \\
\hline Peak Current & I & 100 & A \\
\hline Pulse duration & $\tau$ & 350 & $\mathrm{nS}$ \\
\hline Perveance & & $3.16 \cdot 10^{-6}$ & $\mathrm{~A} / \mathrm{V}^{1.5}$ \\
\hline Energy per shot & & 3.5 & Joule \\
\hline Charge per shot & & 35 & $\mu \mathrm{C}$ \\
\hline Electrons per shot & & $2.2 \cdot 10^{14}$ & particle \\
\hline Beam outer radius & - & 0.42 & $\mathrm{~cm}$ \\
\hline Beam inner radius & & 0.21 & $\mathrm{~cm}$ \\
\hline Beam cross section & & 0.416 & $\mathrm{~cm}^{2}$ \\
\hline Peak Current density & & 240 & $\mathrm{~A} / \mathrm{cm}^{2}$ \\
\hline Peak Power density & & 24 & $\mathrm{MW} / \mathrm{cm}^{2}$ \\
\hline Energy density & & 8.4 & $\mathrm{~J} / \mathrm{cm}^{2}$ per shot \\
\hline Electron density & & $5.25 \cdot 10^{14}$ & $1 / \mathrm{cm}^{2}$ per shot \\
\hline Electron energy density & $\mathrm{E}$ & $5.25 \cdot 10^{13}$ & $\mathrm{MeV} / \mathrm{cm}^{2}$ per shot \\
\hline
\end{tabular}

Note these data are maximum values. Lower values must be applied during experiment and thus the monitor must also be workable at those lower values.

\section{VARIOUS METHODS}

Many methods have been investigated to see if they fit our need. They all have some advantages but are suited only for certain conditions. Following brief descriptions give general ideas for reference.

\section{Normal phosphorescent screen}

Phosphorescent screen has been widely used for imagery, but usually limited to low beam current and low voltage. Beam voltage used in image tubes is normally no more than $20 \mathrm{kV}$. Some accelerator beam profile monitors also use phosphorescent screen, its energy being so high that it penetrates the screen with only a little loss.

SLAC did an in depth study on the phosphorescent screen ${ }^{\text {[2] }}$ of different manufacturers and colors about their linearity, uniformity, low radiation damage and a suitable emitted wavelength for use with television camera. Most of the screens were produced by settling powdered phosphor onto aluminum substrate with a thickness of 0.016 in. $(0.4 \mathrm{~mm})$ or so. The deposited phosphor-binder layer was approximately 0.0035 inches $(0.09 \mathrm{~mm})$ thick. The screen was tilted with the beam axis and perpendicular to the camera axis so as to watch the beam profile. The screen resolution is better than 80 microns. Their record showed a maximum beam intensity of $10^{10}$ particles per pulse. Life tests were up to total $5 \times 10^{15}$ electrons passing through the screen. Some screens were damaged.

It is considered that phosphorescent screens are suited for a modest beam inten- 
sity. Its energy seems not critical providing it can penetrate the screen. The latter is not the case if beam energy only $100 \mathrm{keV}$ or so.

\section{Physical mark image}

Burn-hole. In VLEPP, ${ }^{[3]}$ thin $\mathrm{Al}$ and $\mathrm{Pb}$ films were used to study the focusing for the $14 \mathrm{GHz}$ klystrons. The films were placed inside the beam channel in a series specified locations. The value of expositions (number of shots) was defined at the condition to burn a hole at the filrn and then the subsequent beam would pass through without loss. Thus one could check the variation of the beam cross-section along the channel, see Fig. 1.
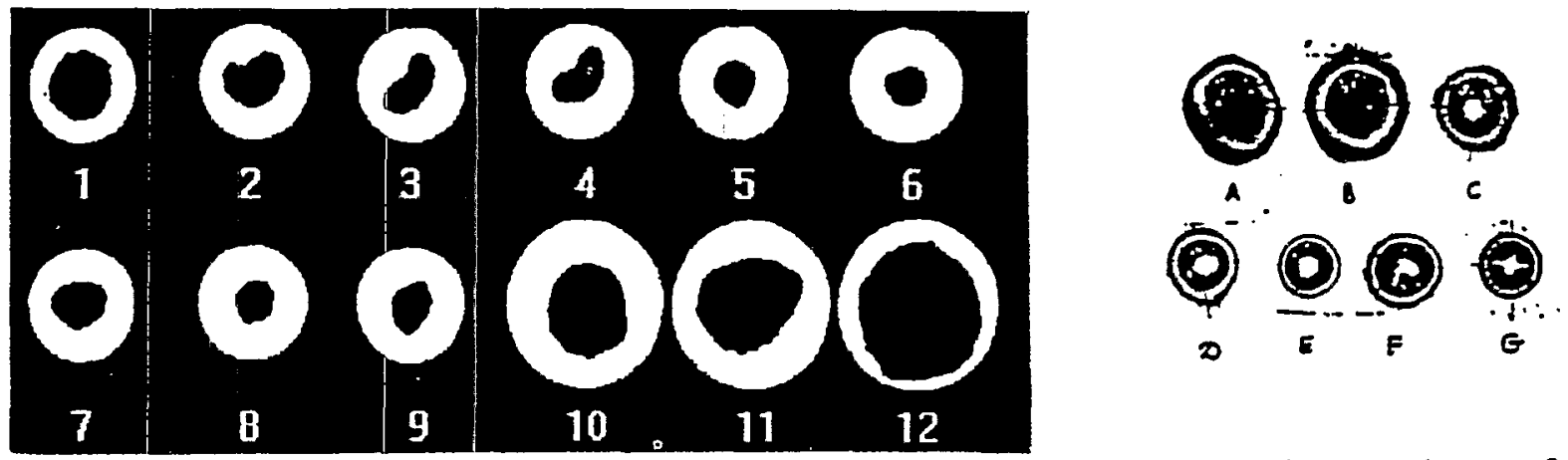

Fig.1 Burn hole as marks of beam shapes.

Fig. 2 The images of partly burnt film

Viniprose film. A similar method was also used in Tomsk Polytechnic University, Russian ${ }^{[4][5]}$. Instead of metal film they used viniprose film, which is coviny chlorid with the methyl methacrylate. see Fig. 2. The center part was burnt, (the center white part in the Figure), the rest part was not completely burnt but the color was changed that the more dark spaces conforms more current density. However, polygraphic opportunities are inhibitory represent exact correspondence between copy and original. The kinetic energy was 240 to $400 \mathrm{kV}$ and $200 \mathrm{~A}$ with $90 \mathrm{~ns}$.

In Sandia National Laboratory, ${ }^{[6]}$ people studied the shape of beam with very strong current and relativistic energy, some $400 \mathrm{kV}$ and $6-16 \mathrm{kA}$, by putting something in the beam path as a "witness plate". Beam hitting these materials will made physical marks on them. They have used graphite plate, brass plate and cellophane.

Graphite plate. The graphite is no less than $1 \mathrm{~cm}$ thick, and thus fully stops the beam. The beam leaves a burnt mark on it.

Brass plate. The beam ablates the surface of the smooth brass and leaves an image on it.

Blue cellophane film. The film is only 0.005 in $(0.127 \mathrm{~mm})$ thick so the beam can pass through it. As it passes through, it deposits a small amount of energy which discolors the blue color leaving a mark of beam profile.

All above methods are destructive, and the witness plate (or films) have to be taken out from the vacuum chamber after testing. The marks are integrated information, can not be watched continuously. The burn-hole method is, of course, not applicable for a hollow beam. 


\section{Transition radiation and Cherenkov light image}

A particle moving at a constant velocity crossing a boundary between two materials with different dielectric constants will produce transition radiation. A high speed particle moving in a dielectric, of which the light speed is less than the speed of the particle, will produce Cherenkov radiation. The transition radiation is also considered as a second order Cherenkov effect. Both radiations may be used as diagnostic tools.

Watski $^{[7][8]}$ and his collaborators first considered the transition radiation in the optical range (optical transition radiation -- or OTR for short) as a diagnostic tool for relativistic beam. They worked on the beam with energy in the 30 to $72 \mathrm{MeV}$ range. They demonstrated the radiation and its angular dependence. They used metal films (aluminum, gold or silver) deposited on Mylar foils.

It was found that the images produced from OTR were significantly sharper than the images produced from phosphor screen.

Castellano $^{[9]}$ et. al. studied the OTR of a beam at energy as low as $1 \mathrm{MeV}$ and showed its effectiveness as a diagnostic tool for long pulse beam.

$\mathrm{ATF}^{[10]}$ has studied OTR for use in the FEL experiments to diagnose the energy and the emittance of the electron beam as well as to image the beam profile. The energy they dealt with was about $4 \mathrm{MeV}(\gamma \approx 9)$. The transition radiation was spread over a large angle.

In $\mathrm{SNL}^{[6]}$, the Cherenkov radiation was made use of to get time resolved information of a beam of their relativistic klystron. A piece of quartz was struck by the beam of about $400 \mathrm{kV}$ and 5 to $20 \mathrm{kA}$ in pulse. The image of the radiation was received either by a fast gated micro-channel plate camera or a streak camera. In NSLS, Yu ${ }^{[11]}$ also tried to use quartz to observe the OTR to get time resolution.

The property of almost instantaneous emission of OTR and Cherenkov radiation makes it possible to monitor the beam profile continuously. The property of energy dependence of OTR may also be used to monitor the energy variation along a macropulse with a rather high sensitivity.

However, it is recognized that the intensity of OTR is very much dependent on the incident beam energy. A beam of only $100 \mathrm{kV}$ is considered too low to produce enough radiation. The Cherenkov effect seems too weak to work either, because the velocity of a $100 \mathrm{kV}$ beam is only 0.55 of light velocity, slightly higher than light velocity in quartz.

\section{Infra-red and visible light image}

One way to monitor the beam shape is to use a thin foil of metal or carbon located in the path of a beam, which passes through the foil with some energy lost on it and heats it up with illumination. The Tandem Operation Group ${ }^{[12]}$ can make many kinds of foils with different materials and thicknesses, the thinnest is less than one micron.

This is considered workable. The difference in our case is that we should not let 
the beam pass through but stop completely. The foil will not be too thin but the energy dynamic range is of concern. A detailed analysis will be described later.

\section{X-ray irnage}

The phosphorescent material (usually being powdered) is not durable under bombardment of a strong beam. It is considered that a refractory material can stand the bombardment and meanwhile produce the X-ray at every point where the electrons hit. Its $\mathrm{X}$-ray image is a good representation of the beam profile.

We should define the term "X-ray image". For a medical X-ray diagnostic or a material inspection by X-ray, the obtained image is from a point X-ray source. The Xray always goes along straight lines without any focus as used in a normal camera. In our case, concerned is the image of the X-ray source itself.

Is an X-ray camera commercially available? The zone plate is of most interest for $X$-ray focusing and under active development. To date the spacial resolution of zone plates for hard X-ray application is about half micron and focusing efficiency about $30 \%$. But most zone plates are designed for applications in 8 to $25 \mathrm{keV}$ X-ray photon range, though higher energy plates can be developed. in addition, a zone plate is chromatic and a monochromator is required ${ }^{[13]}$.

In our case, the X-ray photon energy is up to $100 \mathrm{keV}$, and the spectrum is rather flat below this maximum. Thus a zone plate is not realistic.

A pin-hole X-ray camera, on the other hand, is considered much simpler and realizable. The spacial resolution is not critical in our case and can be arranged at rather short source-optical distance, and the problem of diffraction blurring is negligible ${ }^{[13]}$. However, the pin-hole camera has very low optical efficiency. Some laboratories like SLAC have experience using pin-hole X-ray images as a diagnostic of beam interception but took minutes to get one picture. It may be no problem in a very strong beam struck. as in the present case, but an intensifier is necessary. The quantitative estimate will be discussed later.

\section{Converted image with a beryllium foil}

R. Palmer suggested a scheme that a screen made of beryllium with phosphorescent material on the back. When the beam hits the beryllium, the produced X-ray will excite the phosphorescent material and convert the $\mathrm{X}$-ray image to visible light image. The unique characters of the beryllium are its low specific gravity and high specific heat. The former implies a long particle stop range, the latter combining with a long stop range imply a lower temperature increment. The phosphorescent on the back will prevent the bombardment of the electrons. This is an interesting scheme. The numerical analysis shown later justifies the idea. The concerns are if the phosphorescent material is usable at high temperature, and if the phosphor will saturate and get poor contrast of the image. The toxicity of beryllium is another concern.

\section{Wire net or sweeper}

To monitor the profile of accelerator beams, sometimes a wire is used. ${ }^{[12,14]} \mathrm{A}$ wire is swept across the beam and intercepts a certain amount of current, the detected signals as a function of angle can be processed to develop the profile. Tandem Van-deGraff group has successfully used it to monitor the multi-MeV beam profile. 
Thin wire net or matrix can also used for monitor particle trace without sweeping. In principle, a matrix of insulated wires intercepting current is able to develop the profile of the beam. However, it is limited to solid beams only. The information obtained from a pair of two perpendicular array of wires is not adequate to determine a profile of a hollow beam designed for a cluster klystron.

The net is sometines called "Harps", that is formed by tungsten alloy wires stretched across the beam line. It can stand very high beam intensities. However, the size is usually rather large. It would be difficult to make small enough to fit our need.

\section{Another scheme}

Another method is to put a poloroid film on the path of the beam and then check its image. For a low current beam this may be workable, but very unlikely workable in our case.

\section{HEATING PROBLEMS}

\section{Heat transport capability}

Now that the beam can not pass through the screen, then a question is: can we increase the thickness of the screen to avoid overheat? Let's estimate the heat transport capability. Assume heat transfer through the thickness only. The transport heat power along the axis $z$ can be expressed as:

$$
P=K S \frac{\partial T}{\partial z}
$$

where $T$ is the temperature, $K$ the thermoconductivity and $S$ the cross-section area. For aluminum $K$ is temperature dependent as shown below:

$\begin{array}{ccc}\text { unit } & \mathrm{cal} /{ }^{\circ} \mathrm{C} . \mathrm{cm} . \mathrm{sec} & \text { Watt } /{ }^{\circ} \mathrm{C} . \mathrm{cm} \\ 20^{\circ} \mathrm{C} & 0.57 & 2.4 \\ 300^{\circ} \mathrm{C} & 0.64 & 2.7 \\ 400^{\circ} \mathrm{C} & 0.76 & 3.2 \\ 600^{\circ} \mathrm{C} & 1.01 & 4.2\end{array}$

Substituting the power density in question and the maximum thermoconductivity, $4.2 \mathrm{~W} /{ }^{\circ} \mathrm{C} . \mathrm{cm}$, we get the temperature gradient to be $5.67 \times 10^{6}{ }^{\circ} \mathrm{C} / \mathrm{cm}$ or $567^{\circ} \mathrm{C} / \mu \mathrm{m}$. Certainly this gradient is too high for aluminum. It means the power density is too high to be dissipated by conductivity, even for those materials, of which the conductivity is better than aluminum.

Therefore, we can conclude that the heat stays in the volume where the electrons transfer their energy within the pulse period. Increasing the thickness won't help.

\section{Stop range}

Now that the heat can not dissipate instantly, the second question is: what is the volume which absorbs the beam energy? Then we have to know the depth that a beam 
can pass through, i.e. the stop range.

The definition of the "stop range" of an electron is the distance that the electron travels before coming to rest. Usually it is measured by $\mathrm{g} / \mathrm{cm}^{2}$, the mass in gram per unit area, because the range is not sensitive with materials but depends on the mass it penetrates. Nevertheless, there is difference between high $Z$ (atomic number) and low $Z$ materials.

For an electron beam with energy of $100 \mathrm{KeV}^{1}$, the range for heavy material is ${ }^{[15](a)}$ :

$$
R=0.03 \mathrm{~g} / \mathrm{cm}^{2}
$$

For low- $Z$ material and $0.01 \leq T \leq 2.5 \mathrm{MeV}$, an empirical equation is ${ }^{[15](b)}$ :

$$
R=0.412 T^{1.27-0.0954 \ln T}
$$

where $R$ is in $g / \mathrm{cm}^{2}, T$ is the kinetic energy in $\mathrm{MeV}$. For $T=0.1 \mathrm{MeV}$, it figures out ${ }^{2}$

$$
R=0.0133 \mathrm{~g} / \mathrm{cm}^{2}
$$

The stop rage in $\mathrm{cm}$ is:

$$
\delta=R / p
$$

where $\rho$ is specific gravity in $\mathrm{g} / \mathrm{cm}^{3}$.

\section{Temperature increment}

Assuming the specific heat is constant, and the beam energy loss is uniform along its range, and omitting the heat dissipation during the pulse (350 ns), the instant temperature increment, averaged ${ }^{3}$ in the volume, due to a single pulse is:

$$
\Delta T=\frac{E}{C \rho \delta}=\frac{E}{C R}
$$

where $C$ is specific heat, $E$ is energy density in $J / \mathrm{cm}^{2}$. Substituting $E=8.4 \mathrm{~J} / \mathrm{cm}^{2}$ from Table 1, the temperature increment was calculated as shown in Table.2. If the heat can not be dissipated completely before next pulse comes, the temperature increment will be accumulated by a certain amount (see analysis later). That makes things worse.

Table 2 shows the beam stop range of different materials with the beam energy of $100 \mathrm{KeV}$, as well as those parameters like specific gravity, specific heat, thermal conductivity. Note that all those parameters are not exact constants, they normally change with temperature. Nevertheless it is a reasonable approximation.

As is shown, the instant temperature increment of aluminum can be $700^{\circ} \mathrm{C}$, so it will melt. On the other hand, as expected, the temperature increment of beryllium is

1. For other energy, see Appendix Fig. A-1

2. There are some discrepancy between this empirical formula and calculated table, e.g. [16], published in 1965 , which gives a little optimistic results. We are not sure which is more precise. Note this is only an estimate, the discrepancy will not make significant change on the conclusion.

3. The average means omitting the non-uniformity. 
Tablle 2: Beam stop range in different material

\begin{tabular}{|l|c|c||c|c|c|c|c|}
\hline \multicolumn{2}{|c||}{ Material } & \multirow{2}{*}{$\mathrm{Al}$} & $\mathrm{C}$ & $\mathrm{Be}$ & $\mathrm{W}$ & $\mathrm{Ta}$ \\
\hline & Symb & Unit & & & & & \\
\hline \hline Atomic number & $\mathrm{Z}$ & & 13 & 6 & 4 & 74 & 73 \\
\hline Specific Gravity & $\rho$ & $\mathrm{g} / \mathrm{cm}^{3}$ & 2.7 & $\approx 2$ & 1.848 & 19.3 & 16.6 \\
\hline Specific Heat & $\mathrm{C}$ & $\mathrm{J} / \mathrm{g} \mathrm{K}$ & 0.9 & 0.712 & 1.825 & 0.133 & 0.14 \\
\hline Thermoconductivity & $\mathrm{K}$ & $\mathrm{W} / \mathrm{m} \mathrm{K}$ & 237 & $80-240$ & 201 & 173 & 57.5 \\
\hline Melt point & & ${ }^{\circ} \mathrm{C}$ & 660 & 3650 & .1278 & 3410 & 2996 \\
\hline Mass stop range & $\mathrm{R}$ & $\mathrm{g} / \mathrm{cm}^{2}$ & 0.0133 & 0.0133 & 0.0133 & 0.03 & 0.03 \\
\hline Stop range & $\delta$ & $\mu \mathrm{m}$ & 49 & 67 & 72 & 16 & 18 \\
\hline $\begin{array}{l}\text { Instant temp. incre- } \\
\text { ment }\end{array}$ & $\Delta \mathrm{T}$ & ${ }^{\circ} \mathrm{C}$ & 700 & 884 & 345 & 2105 & 2000 \\
\hline
\end{tabular}

much lower than the others, only $345^{\circ} \mathrm{C}$, much less than the melting point. The refractory materials can also stand the temperature increment.

\section{IMAGE RESOLUTION AND MAXIMUM REPETITION RATE}

It is of concern that if the heat dissipation is too fast, the edge of the temperature image will be smeared during the camera shutter time, meaning the resolution is poor. On the other hand, if the heat dissipation is too slow, the temperature remains pretty high when the next pulse comes, the images of two pulses overlap and may cause overheat. It means the repetition rate is limited. Therefore, we have to check quantitatively the heat dissipation rate.

The heat dissipation equation is:

$$
\frac{\partial T}{\partial t}=\frac{1}{C_{\rho}}\left[K \nabla^{2} T-L_{R}(T)\right]
$$

The first term on the RHS is the contribution due to heat conduction, while the second term is due to radiation. Where $C$ is the specific heat, and $\rho$ specific gravity, $\mathrm{K}$ conductivity coefficient. We assume they are constants for simplicity. $L_{R}$ is a radiation loss parameter with

$$
L_{R}(T)=\frac{2 R}{\delta}
$$

where $\delta$ is the thickness of the foil, and $R$ is the radiation-temperature dependence with

$$
R=\varepsilon \sigma T^{4}
$$

where $T$ is the Kelvin temperature, $\varepsilon$ is the emissivity, and $\sigma$ is the radiation coefficient of black body: 


$$
\sigma=5.670 \times 10^{-8} \mathrm{~W} / \mathrm{m}^{2} \cdot \mathrm{K}^{4}=5.670 \times 10^{-12} \mathrm{~W} / \mathrm{cm}^{2} \cdot \mathrm{K}^{4}
$$

For simplicity, let's consider those contributions separately.

\section{Heat dissipation due to conductivity}

At first, we omit the radiation loss, then the above equation becomes the standard heat-conduction equation.

$$
\frac{\partial T}{\partial t}=a \nabla^{2} T
$$

where $\quad a=\frac{K}{C \rho} \quad$ is considered to be constant.

For the case of a foil, we assume cylindrical symmetry, and that at the edige of the foil is a heat sink with constant temperature of $300 \mathrm{~K}$, or room temperature, and the initial temperature distribution is a rectangular function that the inner part (beam spot, assuming $\mathrm{r}<0.5 \mathrm{~cm}$ ) is at an uniform temperature of $\mathrm{T}_{0}$, while the rest is at the same temperature as that of the heat sink. Then the above equation is:

$$
\frac{\partial T}{\partial t}=a\left[\frac{1}{r} \frac{\partial T}{\partial r}+\frac{\partial^{2} T}{\partial r^{2}}\right]
$$

with initial condition:

$$
T(r, 0)= \begin{cases}T_{0} & 0 \leq \rho \leq 0.5 \\ 300 & 0.5<r \leq R_{\max }\end{cases}
$$

and boundary condition: $\quad T\left(R_{\max }, t\right)=300$ (unit of temperature is $K$ )

For normalization, using the following conversion:

$$
u=\frac{T-300}{T_{0}-300} \quad \rho=\frac{r}{R_{\max }} \quad \tau=\frac{a t}{R_{\text {max }}^{2}}
$$

we get: $\quad \frac{\partial u}{\partial \tau}=\frac{1}{\rho} \frac{\partial u}{\partial \rho}+\frac{\partial^{2} u}{\partial \rho^{2}}$

and

$$
\begin{aligned}
& u(\rho, 0)= \begin{cases}1 & 0 \leq \rho \leq 0.5 \\
0 & 0.5<\rho \leq 1\end{cases} \\
& u(1, \tau)=0
\end{aligned}
$$

The solution is:

$$
\begin{gathered}
u(\rho, \tau)=\sum_{m=1}^{\infty} C_{m} e^{-\mu_{m}^{2} \tau} J_{0}\left(\mu_{m} \rho\right) \\
C_{m}=\left(\int_{0}^{0.5} J_{0}(\rho) \rho d \rho\right) / \frac{1}{2}\left[J_{1}\left(\mu_{m}\right)\right]^{2}
\end{gathered}
$$


$J_{0}$ and $J_{1}$ are the zero and first order Bessel functions;

$\mu_{m}$ is the $\mathrm{m}$-th root of zero order Bessel function;

Fig. 3 shows the numerical results vie computer. ${ }^{[17]}$

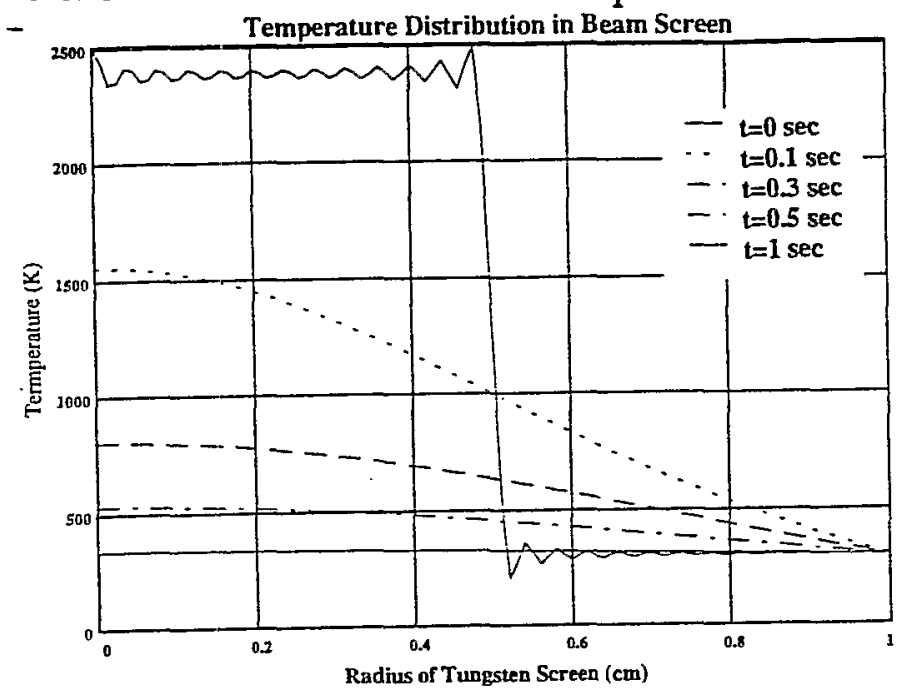

Fig. 3 The temperature distribution at different times

Table. 3 shows the parameter $a$ and $\mathrm{T}_{0}$ for different materials

Table 3:

\begin{tabular}{|l|l||c|c|c|c|c|}
\hline & Unit & W & C & Al & Be & Ta \\
\hline \hline$a$ & $c m^{2} / s e c$ & 0.67 & $\sim 1$ & 0.98 & 0.6 & 0.25 \\
\hline$T_{0}$ & $K$ & 2378 & 1153 & 973 & 617 & 2273 \\
\hline
\end{tabular}

Substituting the parameters we get the temperature distribution at different times

\section{Heat dissipation due to radiation}

Omitting the first term on RHS of (1) and substituting (2) and (3), we get:

$$
\frac{\partial T}{\partial t}=-b T^{4}
$$

where

$$
b=\frac{2 \varepsilon \sigma}{C \rho \delta} \quad \text { is again considered to be constant. }
$$

The solution of Equation (11) is:

$$
t=\frac{1}{3 b T_{0}^{3}}\left(\frac{T_{0}^{3}}{T^{3}}-1\right)
$$

This indicates the time needed to reduce temperature from $T_{0}$ to $T$. Following table shows the numerical examples. 
Table 4:

\begin{tabular}{|l|l||c|c|c|}
\hline & \multicolumn{1}{|c|}{ Unit } & W & C & Be \\
\hline \hline Foil thickness $\delta$ & $10^{-3} \mathrm{~cm}$ & 1.55 & 7 & 7.25 \\
\hline Initial temp. $T_{0}$ & $K$ & 2378 & 1153 & 617 \\
\hline$b$ & $10^{-10} / \mathrm{sec} K^{3}$ & 2.85 & 1.14 & 0.46 \\
\hline$t\left(T / T_{0}=0.5\right)$ & sec & 0.61 & 13.35 & 214 \\
\hline$t\left(T / T_{0}=0.1\right)$ & sec & 87 & 1905 & 30554 \\
\hline
\end{tabular}

This shows that the temperature reduction due to the radiation is very slow. Therefore we can ignore it in comparison with the conductivity dissipation.

\section{Cumulative effect}

There is a question that a bunch of beam hits the screen will result in a temperature increment and will not drop down to the original temperature before the next pulse comes. The next hit will result in a higher temperature than the former hit, and the temperature will get higher and higher. The question is: will it be eventually overheated?

Assuming $T_{n}$, the temperature above room temperature, is a series as follows (i.e. a function of $n$ ):

$$
\begin{aligned}
& T_{1}, T_{2}, T_{3}, \ldots . T_{n} \cdots \\
& T_{1}=0+\Delta T \\
& T_{2}=q T_{1}+\Delta T \\
& T_{n}=q T_{n-1}+\Delta T
\end{aligned}
$$

$q$ is the drop rate, meaning the percentage of the temperature drop down before next pulse coming. $q<1$.

It is easy to figure out:

$$
\begin{aligned}
& T_{n}=\left(q^{n-1}+q^{n-2}+\ldots q+1\right) \Delta T \\
& \lim _{n \rightarrow \infty} T_{n} \rightarrow \frac{\Delta T}{1-q}
\end{aligned}
$$

Therefore, the final temperature is limited. e.g. $q=0.1, T_{n} \rightarrow 1.11 \Delta T$, i.e. $11 \%$ higher than the first hit. This is acceptable. The parameter $q$ and the relation (14) is a way to check the acceptable maximum repetition rate.

\section{X-RAY IMAGE}

A beam hitting a target will produce X-rays. Every spot hit by electrons is an X-ray source. Thus if a target or a foil is put in the path of the beam, the X-ray image will represent the beam profile. The $\mathrm{X}$-ray image can also be a diagnostic of the locations which 
intercept the beam undesirably. As mentioned before, an X-ray camera is not commercially available so far. A pin-hole camera was considered. Since the beam in question is very strong in power, say $100 \mathrm{kV}$ times 100 ampere, the X-ray emission is much stronger than a common X-ray tube, a pin-hole camera is considered workable, though its receiving efficiency is much less than a normal camera. A structure is shown in Fig. 4. The quantitative analyses are described below.

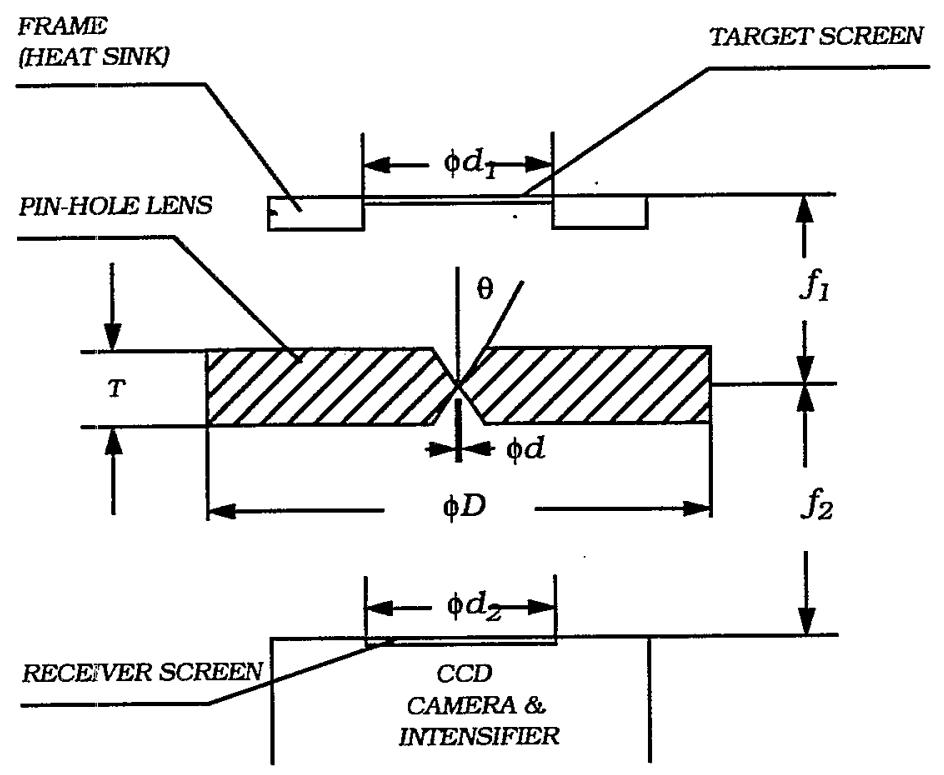

Fig. 4 The structure of a pin-hole camera

\section{The X-ray radiation intensity}

The energy loss of a beam hitting a target includes collision loss and X-ray radiation (i.e. bremstrahlung radiation) loss. For low energy beam, like $100 \mathrm{keV}$, collision loss dominates and eventually is converted to heat, while the radiation loss accounts for only a small part. The radiation yield, $Y$, is defined as the average fraction of its energy that an electron radiates. The average means not only for all particles, but also the radiation of one particle from its initial velocity until completely slowing down. The latter is by no means uniform because a low speed electron has little radiation. It can be calculated according to the following approximate formula ${ }^{1,[15][18] \text { : }}$

$$
Y=\frac{6 \times 10^{-4} \cdot Z T}{1+6 \times 10^{-4} \cdot Z T}
$$

where $Z$ is the atomic number, and $T$ the incident kinetic energy in $\mathrm{MeV}$. In our case the $T$ is rather small, the yleld is almost proportional to the atomic number. So, a high- $Z$ material is preferable if an X-ray image is applied.

The total energy per pulse is the product of the number of electrons and the kinetic energy in electron-volts.

$$
E=5.25 \times 10^{14} \times 100 \mathrm{kV} / \mathrm{cm}^{2}=5.25 \times 10^{13} \mathrm{MeV} / \mathrm{cm}^{2} \text { per pulse }
$$

1. For very low $\mathrm{Z}$, the yield is higher than that by this formula ${ }^{[16]}$, but still in the same order. 
The total radiation energy is $Y E$. It can also be expressed in joules via the conversion formula:

$$
1 \mathrm{MeV}=1.602 \times 10^{-13} \text { joule }
$$

The maximum energy of an X-ray photon is equal to the initial kinetic energy of the incident beam. However, the photon energy spectrum is approximately flat out to this maximum. We assume the average photon energy is $75 \mathrm{KeV}$ or $0.075 \mathrm{MeV}$. Then the photon density radiated from unit area of the target is:

$$
D_{p t}=\mathrm{YE} / 0.075
$$

\section{Receiving intensity in a pin-hole camera}

For a high energy beam the bremstrahlung radiation is emitted mostly in the forward direction. But $100 \mathrm{kV}$ is not a high energy to this point. To be conservative, we assume it radiates uniformly along all $4 \pi$ steradian. If a pin-hole is applied, the radiation intensity at the screen located at the back of the pin-hole will be much reduced. The reduction rate is ${ }^{1}$ :

$$
\alpha=\frac{d^{2}}{16 f_{2}^{2}} \cdot \frac{f_{1}^{2}}{f_{1}^{2}+r_{b}^{2}}
$$

where $d$ is the diameter of the hole, $f_{1}$ is the distance between the hole and the target (radiation source), $f_{2}$ is the distance between the hole and the receiver screen, and $r_{b}$ is the radius of the beam. Assuming $d=0.03 \mathrm{~cm}, r_{b}=0.42 \mathrm{~cm}, f_{1}=2 \mathrm{~cm}, f_{2}=3 \mathrm{~cm}$, then $\alpha=6.0 \times 10^{-6}$. The photon density at the receiver screen $D_{\mathrm{pr}}$, is shown on the 6 th row

\begin{tabular}{|c|c|c|c|c|c|c|}
\hline \multicolumn{3}{|c|}{ Material } & \multirow{2}{*}{$\mathrm{C}$} & \multirow{2}{*}{$\mathrm{Be}$} & \multirow{2}{*}{$\mathrm{Ta}$} & \multirow{2}{*}{ W } \\
\hline & Symbol & Unit & & & & \\
\hline Atomic number & $\bar{Z}$ & & 6 & 4 & 73 & 74 \\
\hline X-ray yield ${ }^{a}$ & $\mathrm{Y}$ & $10^{-3}$ & 0.36 & 2.4 & 0.44 & 4.44 \\
\hline $\mathrm{X}$-ray production ${ }^{\mathrm{b}}$ & YE & $10^{11} \mathrm{MeV} / \mathrm{cm}^{2}$ & 0.19 & 0.13 & 2.3 & 2.3 \\
\hline X-ray energy density & $\overline{\mathrm{D}_{\mathrm{E}}}$ & $10^{-2} \mathrm{~J} / \mathrm{cm}^{2}$ & 0.30 & 0.21 & 3.7 & 3.7 \\
\hline X-ray density at target & $\mathrm{D}_{\mathrm{pt}}$ & $10^{12} \mathrm{p} / \mathrm{cm}^{2}$ & 0.25 & 0.17 & 3.1 & 3.1 \\
\hline $\mathrm{X}$-ray density at receiver & $\mathrm{D}_{\mathrm{pr}}$ & $10^{7} \mathrm{p} / \mathrm{cm}^{2}$ & 0.15 & 0.10 & 1.9 & 1.9 \\
\hline $\begin{array}{l}\text { Photon density after } \\
\text { intensifier }\end{array}$ & & $10^{9} \mathrm{p} / \mathrm{cm}^{2}$ & 0.22 & 0.15 & 2.8 & 2.8 \\
\hline
\end{tabular}
of the Table 5

Table 5:

a. At incident beam energy of $100 \mathrm{keV}=0.1 \mathrm{MeV}$

b. At incident electrons energy density of $\mathrm{E}=5.25 \cdot 10^{13} \mathrm{MeV} / \mathrm{cm}^{2}$.

1. See Appendix B for detail 


\section{Luminosity and camera features}

Now we know the X-ray photon (or quantum) density, the next question is: can it fit a camera, of which the sensitivity is high enough to monitor the beam shape in a single shot and in a real time, or on the contrary, do we have to use a film exposed to many pulses and then develop it?

A camera usually specifies its sensitivity by illuminance and shutter time. The product of them corresponds to the received photon density. The unit of illuminance is Lux, which can be converted to the photon density by formula: ${ }^{1}$

$$
1 \operatorname{lux} \approx 4.1 \times 10^{11} \text { photon } / \text { sec. } \mathrm{cm}^{2}
$$

For example, a PULNiX CCD camera of type TM-745E $\mathrm{E}^{2}$ has features of sensitivity of $0.5 \mathrm{Lux}$ and smearless shutter $1 / 60$ to $1 / 10,000 \mathrm{sec}$. Then the required photon density is:

$$
8.3 \times 10^{-3} \text { to } 5 \times 10^{-5} \text { Lux-sec }
$$

or $\quad 3.4 \times 10^{9}$ to $2.1 \times 10^{7}$ photon $/ \mathrm{cm}^{2}$

Comparing it with Table 5 , it shows that the intensity is in the margin of the camera sensitivity. It is also questionable if the specifications of sensitivity and the fastest shutter time can be satisfied simultaneously. Besides, the specification is for visible light, it will most likely reduce the sensitivity for X-ray. Therefore, an X-ray CCD intensifier is necessary. A DEP Co. (Delft Electronische Producten) intensifier can convert an $\mathrm{X}$-ray quantum into visible light with 150 times photo gain. [19] The last row of the Table 5 shows the results, which is within usable range for a camera. Table 5 shows the data for different materials.

\section{Resolution and edge sharpness}

The definition of the resolution is the minimum distance between two points on the target, that their images will not overlap each other. For a pin-hole camera it is:

$$
\Delta=\left(1+\frac{f_{1}}{f_{2}}\right) d
$$

where $d$ is the diameter of the hole. This can be determined from Fig. 5.

The interception effectiveness for X-rays is determined by the attenuation formulated as:

$$
A_{\mathrm{tt}}=I / I_{O}=e^{-\mu \rho T}
$$

where $p$ is the specific gravity, $T$ is the thickness of the lens. and $\mu$ is the attenuation coefficient, a function of photon energy. For tungsten, $\mu$ equals $5.91 \mathrm{~cm}^{2} / \mathrm{g}$ in $50 \mathrm{keV}$ and $4.43 \mathrm{~cm}^{2} / \mathrm{g}$ in $100 \mathrm{keV}$, but has a minimum $2.49 \mathrm{~cm}^{2} / \mathrm{g}$ in $69.5 \mathrm{keV}$. If we choose

1. 1 Lumen $\approx 1.47 \times 10^{-3}$ Watt @ $\lambda=5.55 \times 10-7 \mathrm{~m}$, the energy of a photon with $\lambda=5.55 \times 10-7 \mathrm{~m}$ is $3.579 \times 10^{-19}$ Joule, therefore 1 Lumen approximately equal $4.1 \times 10^{15}$ photons, and 1 Lux=1Lumen/ $\mathrm{m}^{2}$, so, 1 Lux $\approx 4.1 \times 10^{11}$ photons $/ \mathrm{sec} . \mathrm{cm}^{2}$.

2. That is one we have in hand. 
$T=0.5 \mathrm{~cm}$, then minimum $A_{\mathrm{tt}}$ is $3.67 \times 10^{-11}$. For a tantalum lens it will be $1 \times 10^{-9}$. So the thickness should be adequate for interception in both cases

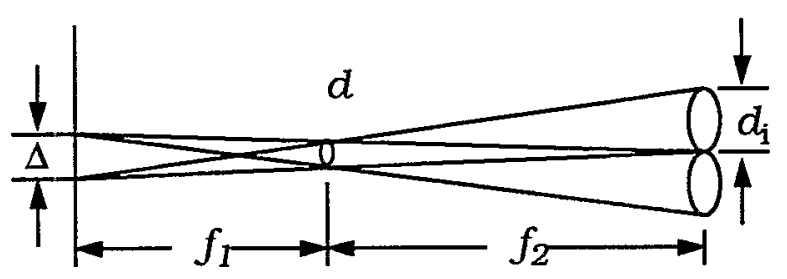

Fig. 5 The resolution for a pin-hole camera

At the edge of the hole, the thickness $t$ increases linearly (see Fig. 4) as:

$$
t=2 x \cot \theta
$$

The edge sharpness can be featured by the differentiation of the attenuation with respect to the distance from the edge, $x$. It is:

$$
\begin{aligned}
& \frac{d A_{t t}}{d x}=-\mu \rho e^{-\mu \rho t} \frac{d t}{d x} \\
& \left.\frac{d A_{t t}}{d x}\right|_{x=0}=-2 \mu \rho \cot \theta=-\frac{1}{\delta}
\end{aligned}
$$

where $\quad \delta=\frac{\tan \theta}{2 \mu \rho} \quad$ and we have $\quad A_{t t}=e^{-\frac{x}{\delta}}$

$\delta$ has the dimension of length, implying that the intensity will decay to $1 / e$ at a distance of $\delta$ from the edge. Substituting $\theta=25^{\circ}$, then for tungsten, $\delta=0.049 \mathrm{~mm}$, for tantalum $\delta=0.056 \mathrm{~mm}$. So, the image edge sharpness should be satisfied. From the above formula, the smaller the span angle $\theta$, the better. But the span angle should be large enough that the image can be viewed from the hole.

\section{ACCKNOWLEDGEMENTS}

The author is, very grateful to many colleagues and friends inside and outside BNL for the very beneficial discussions and information they provided during the investigation. Among them are P. Thieberger, I. Feigenbaum, B. Murray, S. Sengupta, X. J. Wang, T. Srinnivasan-Rao, G. Smith, L. H. Yu, J. W. Wang, D. P. Coleman, D. F. Wang, G. Shea, W. Yun, A. S. Sulakshin, A. N. Sandalov etc. The author would like to extend his thanks also to all colleagues in CAP group, who continuously gave good comments in the group meetings.

\section{REFERENCES}

[1] Y. Zhao, "A proposal for the beam monitor", internal memo, BNL, March, 1996. 
[2] J. T. Seeman, V. Luth, M. Ross, J. Sheppard, "Beam tests of phosphorescent screen", SLAC$\mathrm{CN}-290,1985$

[3] G. V. Dolbilov, et al, "Study of $14 \mathrm{GHz}$ VLEPP klystrons with 11 and $15 \mathrm{~mm}$ aperture", Pulsed RF Sources for Linear Colliders, AIP Conference Proceedings 337, 1994, p.122

[4] F. Isakov, et al, "Status of experiments" on relativistic klystron based on linear induction accelerator", Pulsed RF Sources for Linear Colliders, AIP Conference Proceedings 337, 1994, p. 134

[5] A. S. Sulakshin, private communication

[6] P. D. Coleman, private communication

[7] L. Watski, J. Marcou, and S. Roland "Detection of optical transition radiation and its application to beam diagnostic", IEEE Trans. in Nuclear Science, v. 20, p. 544, 1973.

[8] L. Watski, S. Roland, J. Lasalle, M. Bolore and G. Fillipi, "Interference phenomenon in optical transition radiation and its application to particle beam diagnostics and multiple-scattering measurements", J. Appl. Phys. V. 46,no.8, p.3644, Aug. 1995

[9] M. Castellano et. al., "Analysis of optical transition radiation emitted by a $1 \mathrm{MeV}$ electron beam and its possible use as diagnostic tool", Nucl. Instr. and Meth. in Phys. Res. A, 357, p.231, 1995.

[10] X. Z. Qiu, X. Wang, I. Ben-Zvi, "Proposal for using optical transition radiation for electron beam alignment and emittance measurement for the free electron laser experiments at ATF", BNL Note, 1995.

[11] L. Yu, private communication.

[12] P. Thieberger, I. Feigenbaum, private communication

[13] W. Yun, private communication.

[14] D. F. Wang, private communication

[15] J. E. Turner, "Atoms, radiation, and radiation protection", New York, J. Wiley, 1995. (or BNL Research Library call No.: QC795.T87 1995. )

(a) p.83, Fig. 4.4, (b) p.94 formula 5.16, (c) p.93. Formula 5.14.

[16] M. Berger, S. Seltzer, “Tables of energy-losses and ranges of electrons and positrons", NRC Pub.1133, p.206, 1964

[17] Y. Zhao, H. Wang, "Heat conduction in the beam profile screen for the cluster klystron", internal memo, May, 1996

[18] H. W. Koch and J. W. Motz, Rev. Mod. Phys. 31, 920, 1959

[19] L. A. Bosch, "Dynamic uses of image intensifiers", Delft electronische Producten B.V., P. O. Box $60,9300 \mathrm{AB}$ Roden, The Netherlands, Tech. Note.

[20] S. A. Cherenshchikov et al., "Secondary emission magnetron injection guns current durable electron", Pulsed RF Sources for Linear Colliders, AIP Conference Proceedings 337, 1994, p.350. 
APPENDIX A The ranges of different materials and inject energy

The following figure shows the ranges of electrons, protons and alpha particles in light materials (water, bone and muscle) and heavy material (lead) expressed in $\mathrm{g} / \mathrm{cm}^{2}$. (Quoted from J. E. Turner ${ }^{[15]}$, Fig.4.4, p.83.)

Heavy Charged Particles and Matter

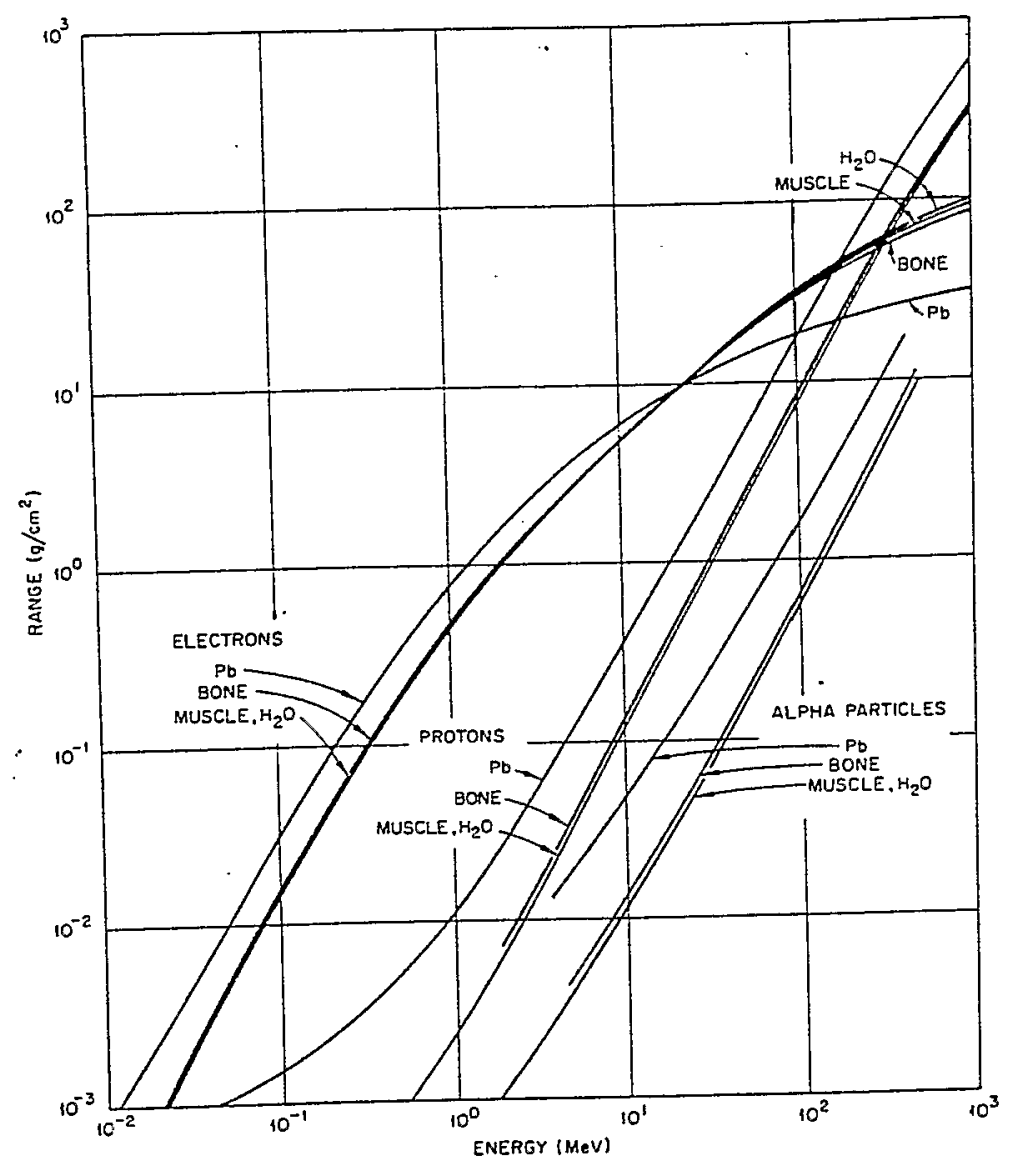




\section{APPENDIX B Pin-Hole Reduction Factor}

Assume: $\Delta S_{1}$ is the cell area of the target, $\Delta S_{2}$ the corresponding image cell area, $f_{1}$ the distance between the target and the pin-hole, $f_{2}$ the distance between the image and the pin-hole, $d$ the diameter of the pin-hole, $\left(\pi d^{2} \ll \Delta S\right), R$ the radiation intensity (total number of photons radiated from unit area, and assume it radiates uniformly along the $4 \pi$ solid angle.)

Then the luminous intensity is:

$$
\Delta S_{1} I_{1} / 4 \pi
$$

The solid angle of the pin-hole is:

$$
\pi d^{2} / 4 f_{1}^{2}
$$

The total number of photons passing through the hole and reaching the $\Delta S_{2}$ is:

$$
\Delta S_{1} I_{1} d^{2} / 16 f_{1}^{2}
$$

Note $\frac{\Delta S_{2}}{\Delta S_{1}}=\frac{f_{1}^{2}}{f_{2}^{2}}$

The luminous intensity at the image is:

$$
I_{2}=\frac{\Delta S_{1}}{\Delta S_{2}} \cdot \frac{I_{1} d^{2}}{16 f_{1}^{2}}=\frac{d^{2}}{16 f_{2}^{2}} I_{1}
$$

So the reduction factor is:

$$
\alpha=\frac{I_{2}}{I_{1}}=\frac{d^{2}}{16 f_{2}^{2}}
$$

and the magnification is $f_{2} / f_{1}$.

At the beam edge, where the distance to the hole is a little larger, the reduction factor will be :

$$
\alpha=\frac{d^{2}}{16 f_{2}^{2}} \cdot \frac{f_{1}^{2}}{f_{1}^{2}+r_{b}^{2}}
$$

where $r_{b}$ is the radius of the beam. 